\title{
Nuclear superfluidity in isospin asymmetric matter within the Skyrme model
}

\author{
R. Aguirre* \\ Departamento de Fisica, Facultad de Ciencias Exactas, Universidad Nacional de La Plata, La Plata, and IFLP, \\ CCT-La Plata CONICET, Argentina
}

(Received 2 February 2012; published 14 June 2012)

\begin{abstract}
The phase diagram of the superfluid phase coupled to spin singlet $(S=0)$ and isospin triplet $(T=1)$ states in infinite nuclear matter is analyzed within the nonrelativistic Skyrme model. We use an approach that allows a unified and consistent treatment of the particle-hole and particle-particle channels. The gap equation is solved for the full range of accessible densities, isospin asymmetries, and temperatures. The characteristic features of each of the components $T_{z}=0, \pm 1$ are emphasized. The search for signatures of a BCS-BEC crossover yields negative results within the scheme proposed.
\end{abstract}

DOI: 10.1103/PhysRevC.85.064314

\section{INTRODUCTION}

The study of the superfluid phase in asymmetric nuclear matter is at present a subject of increasing research [1-7]. It is a matter of intrinsic interest because it explores aspects of the nuclear interaction that are not well established. But it is also a fundamental factor in the description of nuclei in the limits of $\beta$ stability, as well as an important ingredient in the interpretation of the thermal equilibration of protoneutron stars.

In the past, most of the investigations on nuclear superfluidity in infinite matter concentrated on two specific isospin compositions: symmetric matter and neutron matter. Even under the simplifying assumption of homogeneous infinite matter, the study of the superfluid gap requires sophisticated calculations. Starting from a reliable particle-particle potential, the in-medium dressed vertices and the abnormal Green's function must be evaluated self-consistently [8]. Different schemes of approximation have been tried to avoid these involved calculations. Independent efforts focused recently on the formulation of a density functional theory for the pairing interaction that is able to treat the nuclei structure [3-7]. In most of these cases, nuclear matter results are taken as a baseline or as a building block of the approach.

Effective nuclear interactions, such as the Gogny or Skyrme forces, have been profusely used in the context of nuclear superfluidity. The use of effective models, adjusted to reproduce the nuclear phenomenology, offers the advantages of simple calculations and reliable results. In particular, the Skyrme potential motivated different approaches. Since it does not include an explicit pairing interaction, it is frequently used in the particle-hole channel with the addition of an ad hoc particle-particle term [3,7]. However, it is possible to include pairing in the mean-field approach by solving the Hartree-Fock-Bogoliubov equation [9]. In such a way a unified description of the nuclear force is obtained, not only from the conceptual viewpoint but also in the sense that both normal and superfluid properties are evaluated on the same footing.

In this work we intend to take advantage of the properties of the Skyrme model to study the pairing of nucleons coupled

\footnotetext{
*aguirre@fisica.unlp.edu.ar
}

PACS number(s): 21.65.Cd, 21.30.Fe, 21.65.Mn, 21.60.Jz

to spin singlet $(S=0)$ and isospin triplet $(T=1)$ states in infinite nuclear matter. We examine the nuclear phase diagram for the full range of isospin asymmetry, from pure neutron to symmetric nuclear matter, and for temperatures up to the critical value $T_{c}$. In this sense we contribute to complete the panorama of nuclear superfluidity under diverse situations. By choosing the Skyrme model we are able to state the in-medium equations in a simplified way, keeping a unified description of particle-hole and particle-particle channels. To perform the self-consistent solution of the full set of equations we adopt the block diagonalization procedure of Ref. [10].

It is clear that detailed calculations should take into account a multitude of specific effects. However, with the purpose of highlighting the basic features, we present here some simplified calculations, which admit further improvement and completion. For instance, by taking into account that the presence of a pair condensate does not modify significantly the equation of state of infinite nuclear matter [11], it is reasonable to expect the liquid-gas instability to persist under this situation. Inclusion of this effect could modify significatively the superfluid description [12].

This article is organized as follows. In the next section we describe pairing to $S=0, T=1$ states in infinite nuclear matter within the Skyrme model. Results and discussion are given in Sec. III, and finally the conclusions are presented in the last section.

\section{SUPERFLUIDITY IN THE SKYRME MODEL}

The Skyrme model is a well-known effective formulation of the nuclear interaction [13]. It consists of a basic Hamiltonian with contact potentials and density-dependent vertices simulating medium effects:

$$
\begin{aligned}
v_{S k y}\left(r_{1}, r_{2}\right)= & t_{0}\left(1+x_{0} P_{\sigma}\right) \delta\left(r_{1}-r_{2}\right) \\
& +\frac{1}{2} t_{1}\left(1+x_{1} P_{\sigma}\right)\left[\overleftarrow{q}^{2} \delta\left(r_{1}-r_{2}\right)+\vec{q}^{2} \delta\left(r_{1}-r_{2}\right)\right] \\
& +t_{2}\left(1+x_{2} P_{\sigma}\right) \overleftarrow{q} \cdot \delta\left(r_{1}-r_{2}\right) \overleftrightarrow{q} \\
& +\frac{1}{6} t_{3}\left(1+x_{3} P_{\sigma}\right) \delta\left(r_{1}-r_{2}\right) n^{\gamma}\left[\left(r_{1}+r_{2}\right) / 2\right] \\
& +i W_{0}\left(\sigma_{1}+\sigma_{2}\right) \cdot \overleftarrow{q} \times \delta\left(r_{1}-r_{2}\right) \vec{q}
\end{aligned}
$$


where $\sigma_{k}$ represent the Pauli matrices for spin, $P_{\sigma}=\left(1+\sigma_{1}\right.$. $\left.\sigma_{2}\right) / 2$ is the spin exchange operator, $q=-i\left(\nabla_{1}-\nabla_{2}\right) / 2$ is the relative momentum operator, and $n$ is the total baryonic density.

An energy density functional can be obtained by taking the statistical averages of the Hamiltonian

$$
\hat{H}=\sum_{i, j} t_{i, j} a_{i}^{\dagger} a_{j}+\frac{1}{2} \sum_{i, j, k, l} v(i, j ; k, l) a_{i}^{\dagger} a_{j}^{\dagger} a_{k} a_{l}
$$

expanded in a given basis. Here $v(i, j ; k, l)$ stands for the matrix elements of $v_{S k y}\left(r_{1}, r_{2}\right)$ in such a basis. Making use of the Wick theorem at finite temperature [14] and assuming nonzero particle-particle expectation values, one arrives to an energy density functional [10]. For pairs coupled to $S=$ $0, T=1$, the following expression is obtained:

$$
\begin{aligned}
\mathcal{E}= & 4 \int \frac{d^{3} p}{(2 \pi)^{3}} \frac{p^{2}}{2 m} f_{0}(p)+2 \int \frac{d^{3} p}{(2 \pi)^{3}} \int \frac{d^{3} q}{(2 \pi)^{3}} \\
& \times\left[f_{0}(p) U_{0}(p, q) f_{0}(q)+f_{3}(p) U(p, q) f_{3}(q)\right] \\
& +2 \int \frac{d^{3} p}{(2 \pi)^{3}} \int \frac{d^{3} q}{(2 \pi)^{3}} \sum_{k} \alpha_{k} g_{k}^{*}(p) V(p, q) g_{k}(q) .
\end{aligned}
$$

Here $f_{0}$ and $f_{3}$ stand for the statistical occupation factor for the total particle and isotopic asymmetry numbers, respectively. They can be related to the more commonly used occupation numbers $f_{a}(p)$ for protons $(a=1)$ and neutrons $(a=2)$ through $f_{a}=f_{0}+I_{a} f_{3}$ with $I_{a}=(-1)^{a}$. The distribution function for pairs is represented with $g_{k}(p), k=0, \pm 1$, where the subindex is related to the third component of isospin $T_{z}=0, \pm 1$, and $\alpha_{0}=1, \alpha_{ \pm 1}=1 / 2$.

The vertices of the Skyrme model for the different channels are [10]

$$
\begin{aligned}
U_{0}(p, q) & =\left[a_{0}+b_{0}(\mathbf{p}-\mathbf{q})^{2}\right] / 2, \\
U(p, q) & =\left[a_{2}+b_{2}(\mathbf{p}-\mathbf{q})^{2}\right] / 2, \\
V(p, q) & =\left[a_{0}+a_{2}+2 t_{1}\left(1-x_{1}\right)\left(p^{2}+q^{2}\right)\right] / 4,
\end{aligned}
$$

with $\quad a_{0}=6 t_{0}+t_{3} n^{\gamma}, b_{0}=\left[3 t_{1}+t_{2}\left(5+4 x_{2}\right)\right] / 2, a_{2}=$ $-2 t_{0}\left(1+2 x_{0}\right)-t_{3}\left(1+2 x_{3}\right) n^{\gamma} / 3$, and $b_{2}=\left[t_{2}\left(1+2 x_{2}\right)-\right.$ $\left.t_{1}\left(1+2 x_{1}\right)\right] / 2$.

Because we regard proton and neutron numbers as independent conserved charges, different chemical potentials $\mu_{a}$ can be assigned to each one. Alternatively, one can think the total baryonic and isotopic asymmetry numbers are the conserved quantities, with chemical potentials $\mu_{0}$ and $\mu_{3}$, respectively. They are related through $\mu_{a}=\mu_{0}+I_{a} \mu_{3}, a=1,2$. The quasiparticle picture arises from the mutual interaction of the nucleons in the nuclear environment; therefore their properties are unknown until the full problem is solved. However, in the Landau-Fermi liquid approach, one can obtain the energy spectrum in terms of functional derivatives of the energy [10],

$$
2 \varepsilon_{a s}(p)=\frac{\delta \mathcal{E}}{\delta f_{a}(p)}, \quad 2 \alpha_{k} \Delta_{k}(p)=\frac{\delta \mathcal{E}}{\delta g_{k}^{*}(p)},
$$

for $a=1,2, k=0, \pm 1$, where the subindex $s$ distinguishes the two possible spin states, and $\Delta_{k}$ represents the components of the superfluid gap. In such a way we obtain the self-consistent definitions

$$
\begin{gathered}
\varepsilon_{a s}(p)=\frac{p^{2}}{2 m_{a}^{*}}+\frac{1}{8} v_{a}, \\
\Delta_{k}(p)=\frac{1}{(2 \pi)^{3}} \int d^{3} q g_{k}(q) V(p, q),
\end{gathered}
$$

where

$$
\begin{aligned}
v_{a}= & \left(a_{0}+a_{2} w I_{a}\right) n+\sum_{c=1,2}\left(b_{0}+I_{a} I_{c} b_{2}\right) K_{c} \\
& +\left[3-\left(1+2 x_{3}\right) w^{2}\right] \gamma t_{3} n^{\gamma+1} / 6
\end{aligned}
$$

and we used the isospin asymmetry factor $w=\left(n_{2}-n_{1}\right) / n$, the effective nucleon mass

$$
\frac{1}{m_{a}^{*}}=\frac{1}{m}+\frac{1}{4} n\left(b_{0}+b_{2} w I_{a}\right)
$$

and the kinetic term

$$
K_{c}=\frac{2}{(2 \pi)^{3}} \int d^{3} p p^{2} f_{c}(T, p) .
$$

The explicit form of the distributions $f_{0}, f_{3}$, and $g_{k}$ can be obtained in a self-consistent manner by the method of block diagonalization described in Ref. [10]. In order to simplify the discussion we consider two different situations: (i) neutronproton pairs coupled to $T_{z}=0$ and (ii) coupling to $T_{z}= \pm 1$. For these cases we obtain

$$
\begin{aligned}
\text { (i) } f_{0} & =\frac{1}{2}-\frac{\xi_{0}}{4 E_{0}}\left[\tanh \left(\frac{\beta}{2}\left(E_{0}-\xi_{3}\right)\right)+\tanh \left(\frac{\beta}{2}\left(E_{0}+\xi_{3}\right)\right)\right], \\
f_{3} & =-\frac{1}{4}\left[\tanh \left(\frac{\beta}{2}\left(E_{0}+\xi_{3}\right)\right)-\tanh \left(\frac{\beta}{2}\left(E_{0}-\xi_{3}\right)\right)\right], \\
g_{0} & =-\frac{\Delta_{0}}{4 E_{0}}\left[\tanh \left(\frac{\beta}{2}\left(E_{0}-\xi_{3}\right)\right)+\tanh \left(\frac{\beta}{2}\left(E_{0}+\xi_{3}\right)\right)\right], \\
\text { (ii) } f_{0} & =\frac{1}{2}-\left[\frac{\xi_{0}-\xi_{3}}{4 E_{-1}} \tanh \left(\frac{\beta E_{-1}}{2}\right)+\frac{\xi_{0}+\xi_{3}}{4 E_{1}} \tanh \left(\frac{\beta E_{1}}{2}\right)\right], \\
f_{3} & =\frac{\xi_{0}-\xi_{3}}{4 E_{-1}} \tanh \left(\frac{\beta E_{-1}}{2}\right)-\frac{\xi_{0}+\xi_{3}}{4 E_{1}} \tanh \left(\frac{\beta E_{1}}{2}\right), \\
g_{k} & =-\frac{\Delta_{k}}{2 E_{k}} \tanh \left(\frac{\beta E_{k}}{2}\right), k= \pm 1 .
\end{aligned}
$$

Here we used $\xi_{0}=\left(\varepsilon_{2}+\varepsilon_{1}\right) / 2-\mu_{0}, \xi_{3}=\left(\varepsilon_{2}-\varepsilon_{1}\right) / 2-\mu_{3}$, $E_{0}=\sqrt{\Delta_{0}^{2}(p)+\xi_{0}^{2}}$, and $E_{ \pm 1}=\sqrt{\Delta_{k}^{2}(p)+\left(\xi_{0}+k \xi_{3}\right)^{2}}$ for $k= \pm 1$.

The set of self-consistent equations is completed with the relation between the conserved particle numbers and the corresponding chemical potentials,

$$
n_{a}=\frac{2}{(2 \pi)^{3}} \int d^{3} p f_{a}(T, p) .
$$

It must be pointed out that, due to the structure of $V(p, q)$, the momentum dependence of the gap can be expressed as $\Delta_{k}(p)=C_{k}+D_{k} p^{2}$, where $C_{k}$ and $D_{k}$ are functions of the densities and the temperature. 


\section{RESULTS AND DISCUSSION}

For the Skyrme model the SLy4 parametrization is used, for which $t_{0}=-2488.91 \mathrm{MeV} \mathrm{fm}^{3}, t_{1}=486.82 \mathrm{MeV} \mathrm{fm}^{5}, t_{2}=$ $-546.39 \mathrm{MeV} \mathrm{fm}^{5}, t_{3}=13777 \mathrm{MeV} \mathrm{fm}^{7 / 2}, x_{0}=0.834, x_{1}=$ $-0.344, x_{2}=-1, x_{3}=1.354$, and $\gamma=1 / 6$ [15]. The saturation density, binding energy, incompressibility, and symmetry energy obtained are $n_{0}=0.159 \mathrm{fm}^{-3}, E_{B}=-15.97 \mathrm{MeV}$, $K=229.9 \mathrm{MeV}$, and $E_{S}=32 \mathrm{MeV}$, respectively. Another significative quantity is the in-medium nucleon mass at the saturation density, for which $m^{*} / m=0.694$.

As is common in nonrelativistic calculations, we found that the definition of the gap function contains divergent integrals, which must be regularized in order to obtain meaningful results. We adopt here the intuitive procedure of introducing a momentum cutoff. There is no general argument to select this cutoff, which in most calculations is chosen as a compromise between conceptual reasons and computational efficiency. Anyway, it must be regarded as a part of the definition of the model. Actually, for a given number density of particles $n$, we use the cutoff $p_{c}=1.3 p_{F}$, where $p_{F}=\left(3 \pi^{2} n\right)^{1 / 3}$ is the Fermi momentum. Since we plan to examine the low-temperature domain, this is a reasonable prescription which ensures that Fermi surface effects are properly included and the standards used to fix the SLy4 parametrization are well reproduced. We leave for further development a detailed study of the dependence on the factor $p_{c} / p_{F}$.

As a first step we examine the domain of existence of superfluid phases, which corresponds to a volume in the $n-w-T$

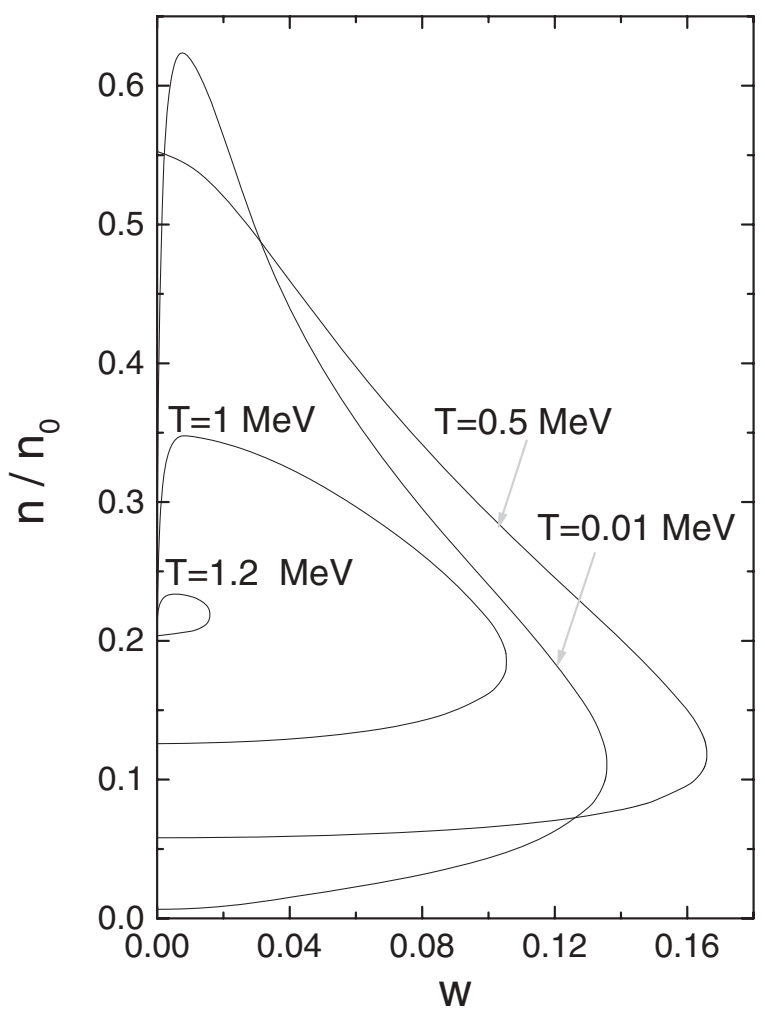

FIG. 1. Isothermal sections of the domain of the neutron-proton coupling. The closed curves are their boundaries; the labels associated with each curve indicate the corresponding temperature.

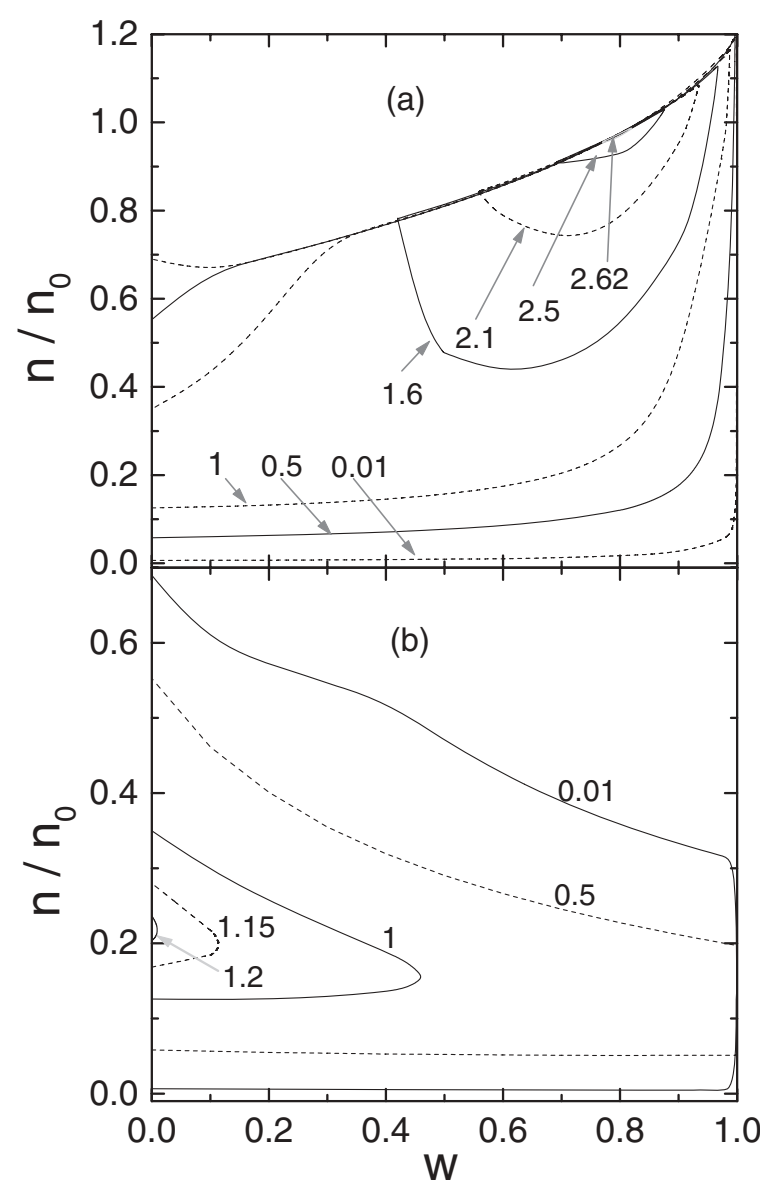

FIG. 2. Isothermal sections of the domain of the proton-proton (a) and neutron-neutron (b) couplings. The closed curves are their boundaries; the labels associated with each curve indicate the corresponding temperature expressed in $\mathrm{MeV}$.

space. The results are shown in Fig. 1 for the neutron-proton coupling $\left(T_{z}=0\right)$ and in Figs. 2 and 3 for the neutron-neutron and proton-proton cases $\left(T_{z}= \pm 1\right)$. Since the $T_{z}=0$ pairing needs a balanced population of neutrons and protons, it arises on a very narrow range of asymmetries, $0 \leqslant w<0.16$, and relatively low densities, $0<n / n_{0}<0.6$. As the temperature is increased above $T=0.5 \mathrm{MeV}$ the domain is reduced, shrinking to a point at the critical temperature $T_{c} \simeq 1.2 \mathrm{MeV}$. As expected, this critical value is reached in symmetric matter.

A contrasting result is obtained for $T_{z}= \pm 1$, for which the full range of asymmetries is covered. There are noticeable differences between neutron-neutron $\left(T_{z}=-1\right)$ and protonproton $\left(T_{z}=1\right)$ couplings. In the first case, displayed in Fig. 2(b), the reach in density is not beyond $n / n_{0} \simeq 0.6$, and the critical temperature is $T_{c} \simeq 1.2 \mathrm{MeV}$. Neutron-proton and neutron-neutron couplings have the same critical temperature because it is attained at $w=0$ in both cases. In symmetric matter neutrons and protons are indistinguishable since we do not include the electromagnetic interaction. For pure neutron matter the gap has collapsed for the curves corresponding to $T>0.5 \mathrm{MeV}$. The proton-proton coupling persists up to supranormal densities $n / n_{0} \simeq 1.2$ under extreme isospin asymmetries $w \simeq 1$ and very low temperatures, as can be seen 


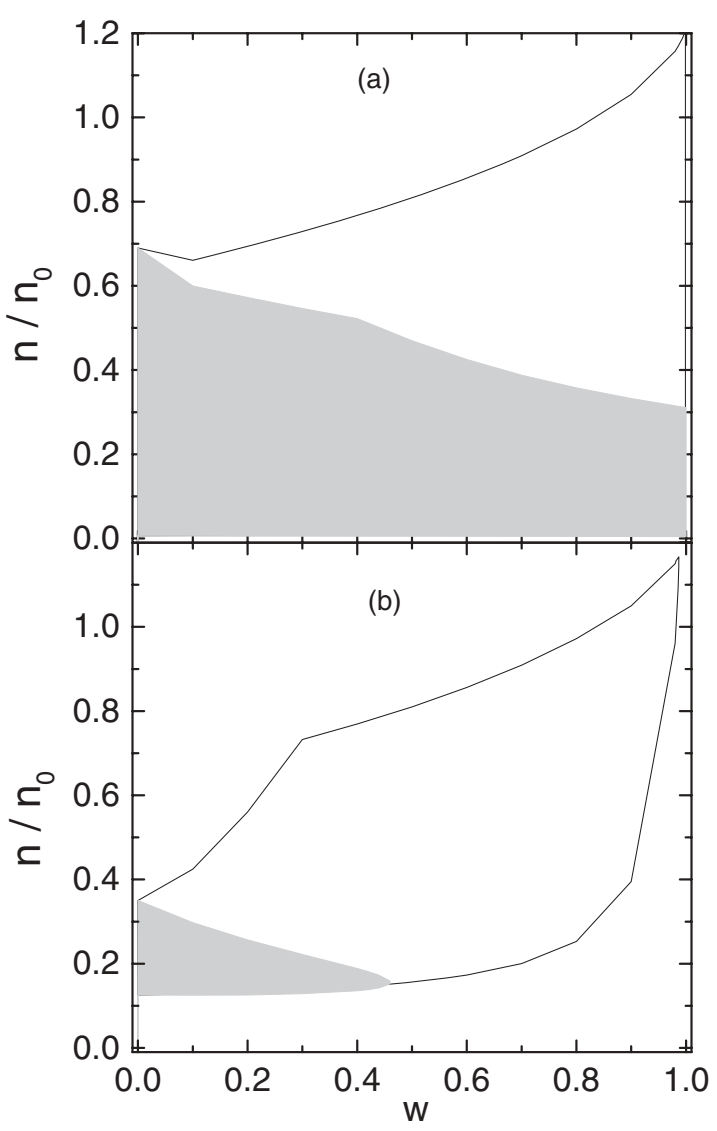

FIG. 3. Detail of the relation between the isothermal sections of the domains of the neutron-neutron and proton-proton couplings for $T=0.01 \mathrm{MeV}$ (a) and $T=1 \mathrm{MeV}$ (b). The shaded areas correspond to the $T_{z}=-1$ component, whereas the closed curves stand for the boundaries of the $T_{z}=1$ region.

in Fig. 2(a). The critical temperature $T_{c} \simeq 2.6 \mathrm{MeV}$ takes place at $w=0.8$. It must be noticed that the gap for $T_{z}=-1$ appears always associated with the $T_{z}=1$ case, as is emphasized in Fig. 3. The shaded area represents the domain of the $T_{z}=-1$ pairing for a selected temperature, while the outer curve shows the limits of the region of existence for the $T_{z}=1$ coupling.

Next we examine the behavior of the gap function evaluated at the Fermi surface. As was mentioned at the end of the previous section, within the Skyrme model the gap has a simple parabolic dependence with the momentum, and therefore it can be written $\Delta_{F k}=\Delta_{k}\left(p_{F}\right)=C_{k}+D_{k} p_{F}^{2}$. In Fig. 4 we display the density dependence of the neutron-proton gap at the Fermi surface for the low temperature $T=0.01 \mathrm{MeV}$ [Fig. 4(a)] and for $T=1 \mathrm{MeV}$ [Fig. 4(b)] close to the critical temperature. We found typical bell-shaped curves, exhibiting the property that the maximum value for $\Delta_{F 0}$, the density where it happens, and the range of existence are all decreasing functions of $w$. For instance, for $w=0$ and $T=0.01 \mathrm{MeV}$ the maximum $\Delta_{F 0}=1.6 \mathrm{MeV}$ corresponds to $n / n_{0} \simeq 0.175$ and the range of densities is $0<n / n_{0}<0.62$. At this temperature neutronproton pairing is not sustained for asymmetries beyond $w>$ 0.12 . For $T=1 \mathrm{MeV}$ the gap has disappeared for the more asymmetric configurations $(w>0.04)$. For a given value of $w$ the maxima have been reduced by around $40 \%-50 \%$ with

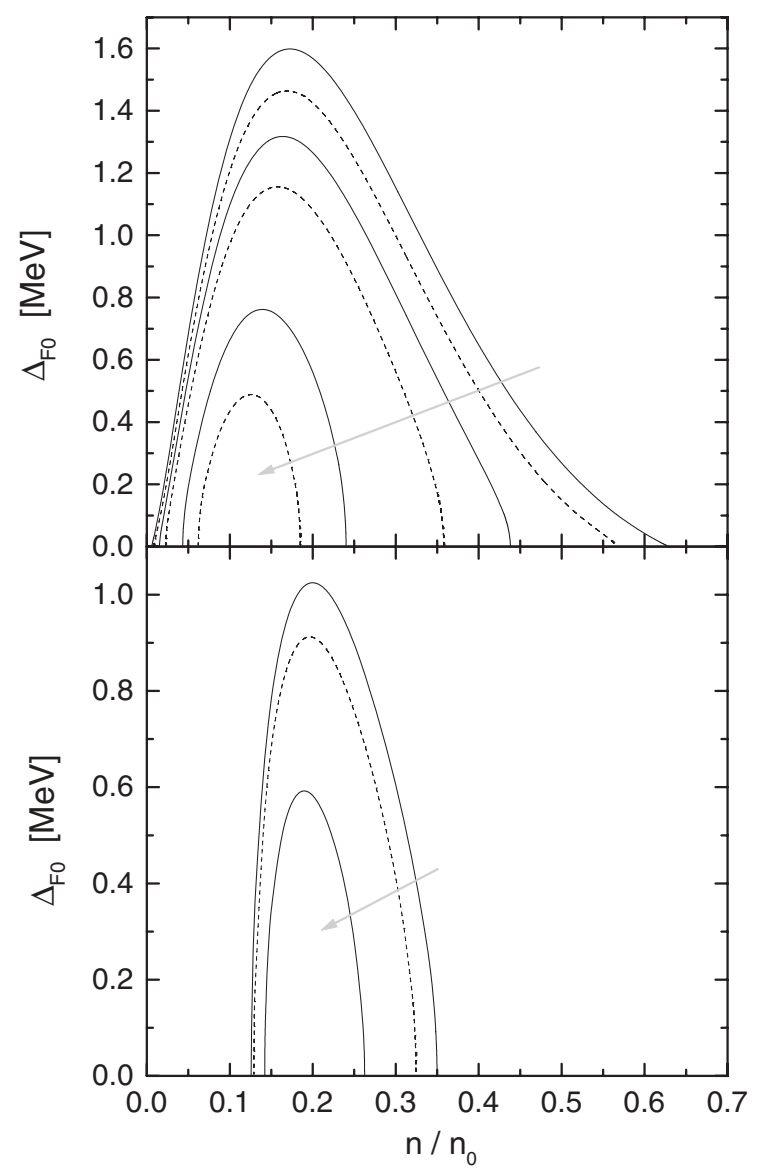

FIG. 4. The density dependence of the neutron-proton gap for several asymmetries and constant temperature $T=0.01 \mathrm{MeV}$ (a) and $T=1 \mathrm{MeV}$ (b). The arrows indicate the direction of growing $w$, taking the specific values $w=0,0.02,0.04,0.06,0.1$, and 0.12 (a) and $w=0,0.02$, and 0.04 (b).

respect to the equivalent case at $T=0.01 \mathrm{MeV}$, and they are located at slightly lower densities.

A similar description for the $T_{z}= \pm 1$ case is exhibited in Figs. 5 and 6. A direct comparison of the last three figures indicates that for $w=0$ all the cases $T_{z}=0, \pm 1$ coincide. This is a direct consequence of the indistinguishability of protons and neutrons for $w=0$, if electromagnetic interactions are neglected. Differences appear as $w$ is increased. While the magnitude of $\Delta_{F 0}$ drops drastically, there is a smooth decrease for neutron-neutron and proton-proton pairings. In the former case the density domain is progressively reduced, keeping its center around $n / n_{0} \sim 0.2$. For $T_{z}=1$, instead, this base becomes broader for increasing $w$. From Fig. 6(a), it can be seen that, for $T_{z}=1$ and $T=1 \mathrm{MeV}$, the maximum value of the gap at the Fermi surface starts increasing for $0<w<0.4$ and from here on it decreases slowly.

Nuclear superfluidity in pure neutron and symmetric nuclear matter has been extensively studied at zero temperature by using a variety of theoretical approaches. We do not intend to reach the same accuracy of the more sophisticated calculations, but a comparison is in order to check the reliability of our outcome. For instance, the maximum value of the neutron gap in neutron matter, $\Delta_{F-1} \simeq 1.4 \mathrm{MeV}$, corresponding to 


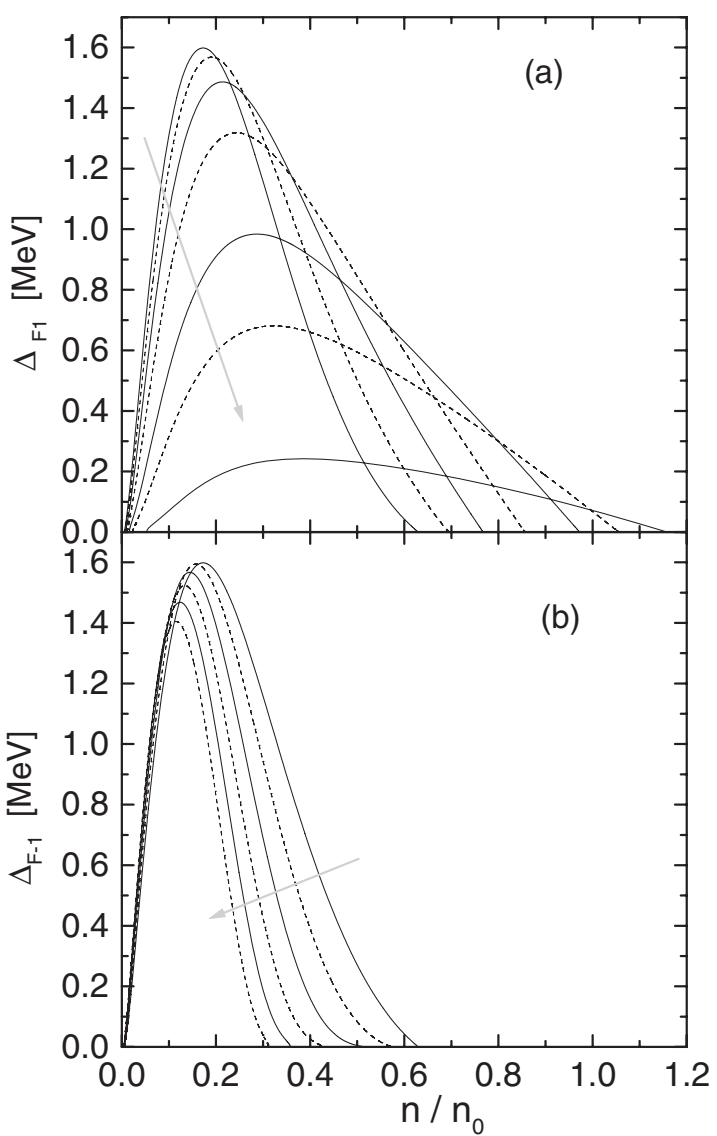

FIG. 5. The density dependence of the $T_{z}=1$ (a) and $T_{z}=-1$ (b) gaps at $T=0.01 \mathrm{MeV}$. The arrows indicate the direction of growing $w$, taking the specific values $w=0,0.2,0.4,0.6,0.8,0.9$, and 0.98 (a) and $w=0,0.2,0.4,0.6,0.8$, and 1 (b).

$n / n_{0}=0.12$ [Fig. 5(b)], is lower than the $2-2.5 \mathrm{MeV}$ obtained in quantum Monte Carlo calculations [16]. Our result seems to be more similar to that of Ref. [12], which gives approximately $1.75 \mathrm{MeV}$ at $n / n_{0} \simeq 0.12$. However, for $w=0$, in the same reference the authors obtain a maximum value of about $2.7 \mathrm{MeV}$ at $n / n_{0} \sim 0.09$. This is in clear contrast with the value $\Delta_{F}=1.6 \mathrm{MeV}$ at $n / n_{0}=0.17$ shown in Fig. 5(a).

With regard to the variation with $w$ shown in Fig. 5, it is useful to make a comparison with Figs. 1 and 2 of Ref. [2]. In this work the superfluid gap is evaluated in a BCS approach, over a Brueckner-Hartree-Fock calculation based on the Argonne AV18 model plus a three-body force. Some of the coincidences we find for the neutron gap are as follows: all the maxima are roughly centered around $n=0.02 \mathrm{fm}^{-3}$, as $w$ increases the maximum slightly shifts toward smaller $n$, and, finally, lesser extinguishing density corresponds to the larger asymmetries. The upper density supporting the neutron gap ranges from $n / n_{0}=0.47$ to 0.70 for $w=1$ and $w=0$, respectively [17]. We have obtained an inferior set of values, $0.3<n / n_{0}<0.65$, for the same instance [see Fig. 5(b)]. A significative difference is the fact that the peak value of $\Delta_{F-1}$ increases with $w$, in opposition to the tendency illustrated in Fig. 5(b). For the proton gap we also find some coincidences for growing $w$ : the peak value of $\Delta_{F 1}$ decreases (in fact a $40 \%$ decrement is registered between $w=0$ and $w=0.8$

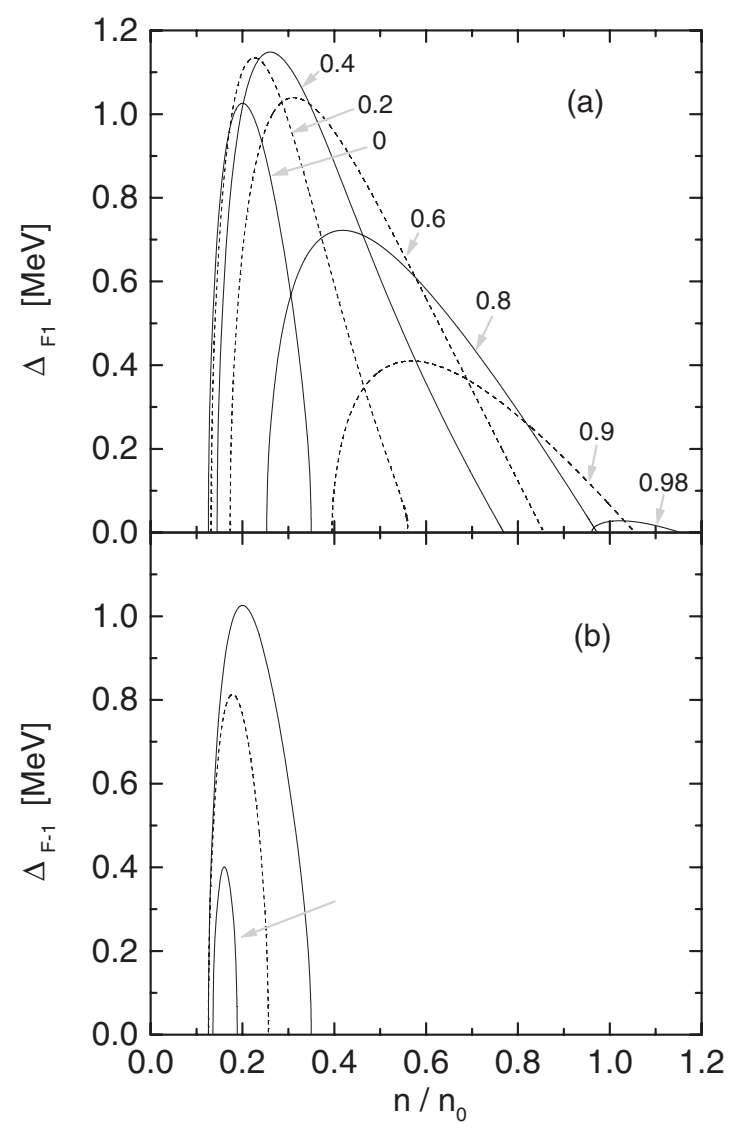

FIG. 6. The density dependence of the $T_{z}=1$ (a) and $T_{z}=-1$ (b) gaps at $T=1 \mathrm{MeV}$, for several asymmetries. The numerical labels in (a) represent the values of $w$, whereas the arrow in (b) shows the direction of growing $w=0,0.2$, and 0.4 .

in both calculations), the position of this peak moves to the right, and the upper density of existence is increased, reaching $n / n_{0}=1.6$ in Ref. [2], in contrast with our result $n / n_{0}=1$. It must be pointed out that the inclusion of three-body forces in Ref. [2] is essential to improve the coincidence with our results.

A detailed study of the dependence of $\Delta_{F k}$ with $w$ is shown in Fig. 7, where the total density $n$ is kept fixed and $T=$ $0.01 \mathrm{MeV}$ [Figs. 7(a) and 7(c)] or $T=1 \mathrm{MeV}$ [Figs. 7(b) and $7(\mathrm{~d})]$. At low temperature $(T=0.01 \mathrm{MeV})$ there is a change of character according to the value selected for $n$. For densities low enough we found a monotonic trend, decreasing for $\Delta_{F 1}$ and increasing for $\Delta_{F-1}$. For intermediate values of $n$ the curves show a nonmonotonic behavior. This tendency is changed again for $\Delta_{F-1}$ with $n / n_{0}=0.25$ and 0.5 , becoming a monotonic decreasing function of $w$. At $T=1 \mathrm{MeV}, \Delta_{F-1}$ is always decreasing, but $\Delta_{F 1}$ exhibits a maximum situated at increasing values of $w$ as $n$ grows.

Figures 7(a) and 7(c) can be compared with the results presented in Fig. 2 of Ref. [6], which uses a BCS approach over a Brueckner-Hartree-Fock evaluation of the AV18 model. Under these conditions a change in the behavior of $\Delta_{F k}$ as a function of $w$ is reported, similar to that described above. The proton gap decreases monotonically if $n / n_{0}<0.25$, and it develops a maximum for larger values of this parameter. These 


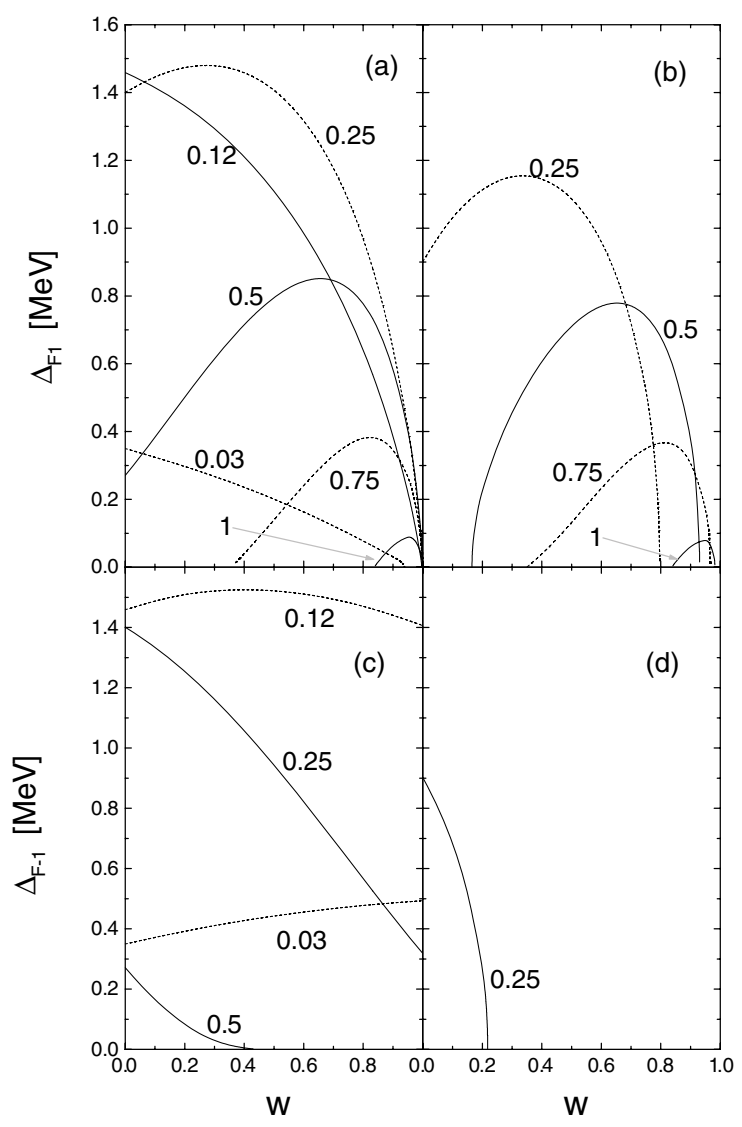

FIG. 7. The dependence on $w$ of the $T_{z}=1[$ (a) and (b)] and $T_{z}=-1[(\mathrm{c})$ and (d)] gaps at $T=0.01 \mathrm{MeV}$ [(a) and (c)] or $T=$ $1 \mathrm{MeV}\left[(\mathrm{b})\right.$ and (d)] for several values of the relative density $n / n_{0}$ as indicated by the numerical labels.

maxima shift toward higher $w$ as $n / n_{0}$ grows. The authors of Ref. [6] found the neutron gap to be an increasing function of $w$ for $n / n_{0}<0.12$, turning decreasing for greater values of the density. There is a strong disagreement in the qualitative description for $n / n_{0}=0.5$, which in our calculations ends at $w \sim 0.4$. Furthermore, absolute values are not comparable since for symmetric matter $1.5 \mathrm{MeV} \leqslant \Delta_{F k}<2.8 \mathrm{MeV}$ is obtained in Ref. [6]. However, it must be noticed that these results are not completely self-consistent.

The thermal dependence of the gaps is examined in Figs. 8 and 9. For $T_{z}=0$ (Fig. 8) and $w=0$ thermal effects are negligible if $T<0.3 \mathrm{MeV}$, and a sudden drop is registered for $T>1.1 \mathrm{MeV}$. In the same figure, but for $w=0.05$ and 0.1 there is, instead, a smooth increase for temperatures below $T=0.4 \mathrm{MeV}$ and a steep fall for $T>0.8 \mathrm{MeV}$. As the asymmetry is increased the gap disappears at very low temperatures, giving rise to two different critical temperatures. For the case shown $(w=0.15)$ we have $T_{c 1}=0.2 \mathrm{MeV}$ and $T_{c 2}=0.6 \mathrm{MeV}$. This phenomenon has been observed in relation to ${ }^{3} S_{1-}{ }^{3} D_{1}$ coupling [19] and is related to an abnormal behavior of the entropy.

In Fig. 9 we display the results for proton [Figs. 9(a) and 9(c)] and neutron [Figs. 9(b) and 9(d)] gaps. They are grouped according to the constant value of the density, $n / n_{0}=0.2$ [Figs. 9(a) and 9(b)] or $n / n_{0}=0.4$ [Figs. 9(c) and 9(d)].

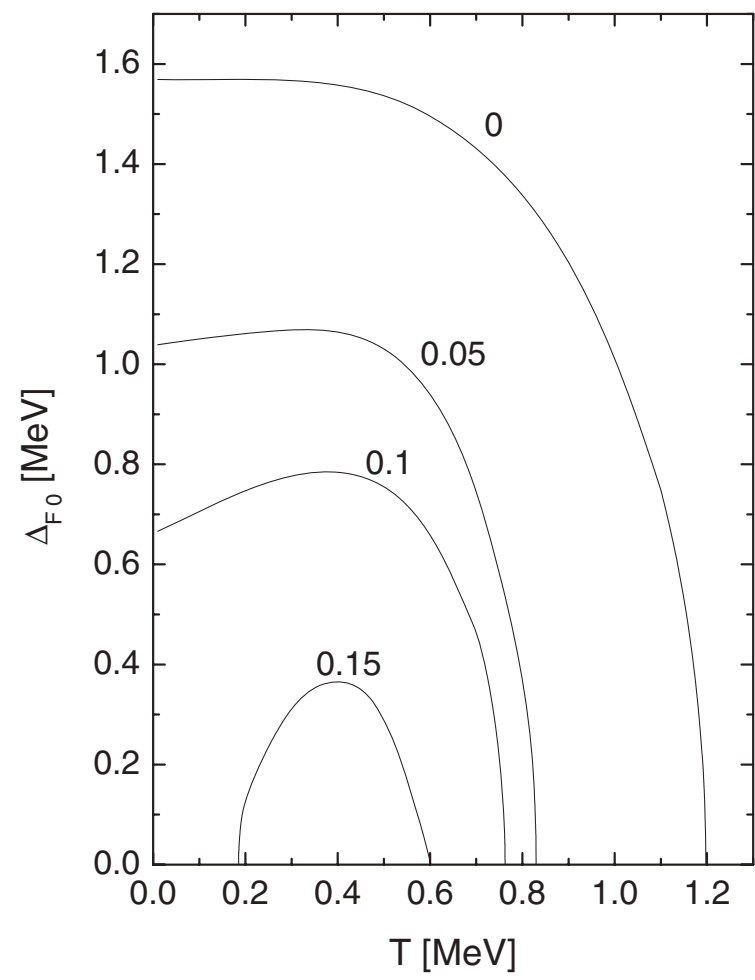

FIG. 8. The thermal dependence of the $T_{z}=0$ gap for $n / n_{0}=0.1$ and several asymmetries as indicated by the numerical labels.

The gap equation, within the Skyrme model, can be expressed in terms of the coefficients $C_{k}$ and $D_{k}$ :

$$
\begin{aligned}
C_{k} & =\frac{1}{8 \pi^{2}} \int d q q^{2} g_{k}(q)\left[a_{0}+a_{2}+2 t_{1}\left(1-x_{1}\right) q^{2}\right] \\
D_{k} & =\frac{1}{4 \pi^{2}} t_{1}\left(1-x_{1}\right) \int d q q^{2} g_{k}(q) .
\end{aligned}
$$

We illustrate their density and temperature dependence in Fig. 10 for a set of of typical values. The range of existence of solutions determines the range of the associated $\Delta_{k}$. Here a mirror-like behavior between $C_{k}$ and $D_{k}$ can be seen. This property, together with the fact that $D_{k}<0$, implies that, for fixed temperature and $w$, the maxima of $\Delta_{F k}$ lies at the left of the peaks of $C_{k}$ and $D_{k}$. Furthermore, the presence of a plateau in Fig. 10(b) and the steep fall shown in this figure are the direct cause of the similar behavior registered in Fig. 8.

As a final application we have studied the possibility of a change of regime in the coupling between nucleons. It is believed that at very low densities the pairing bound becomes stronger, giving rise to Bose-Einstein condensation (BEC). The transition from an ordinary superfluid state to BEC should be a smooth one. Recently, there has been a growing interest in the study of a spin singlet condensate [18]. Different indicators have been proposed to signal this crossover, the most accepted is the change in the sign of the chemical potential. With this motivation, we have examined the effective neutron chemical potential $\mu_{n}-v_{n} / 8$ at zero temperature. We focused on the low-density regime, $n / n_{0}<0.001$, of symmetric nuclear 


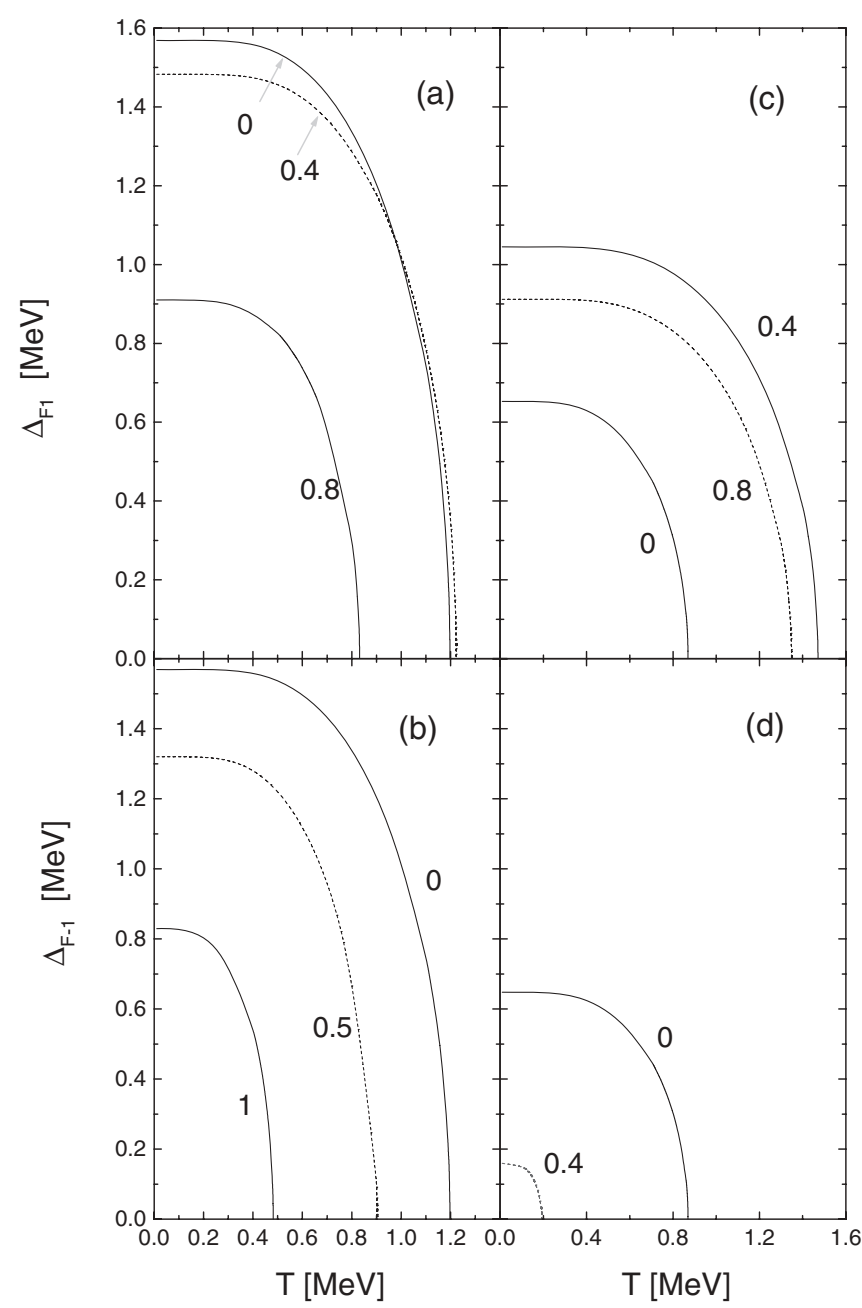

FIG. 9. The thermal dependence of the $T_{z}=1[$ (a) and (c) $]$ and $T_{z}=-1\left[(\mathrm{~b})\right.$ and (d)] gaps at fixed densities $n / n_{0}=0.2[$ [a) and (b)] or $n / n_{0}=0.4$ (c) and (d) and several asymmetries as indicated by the numerical labels.

matter, but we did not find a change of sign. We conclude that the approach used cannot describe the BCS-BEC crossover.

\section{CONCLUSIONS}

In this work we have examined in detail the phase diagram of the $S=0, T=1$ superfluid gap in infinite nuclear matter. We have paid special attention to the thermal and isospin composition effects. For this purpose we have selected the effective Skyrme model of the nuclear interaction. It has been extensively used in diverse fields of nuclear physics with remarkable success. We have used a Hartree-FockBogoliubov-like approach that uses the same interaction as the source for both particle-hole and particle-particle channels. Thus we can give a conceptually unified description of the problem. Furthermore, within our calculations, normal and superfluid properties are taken on the same footing, ensuring the self-consistency of the results.

The neutron-proton component has a very small domain, $n<0.7 n_{0}, w<0.17$, and $T<1.2 \mathrm{MeV}$. The neutron-neutron
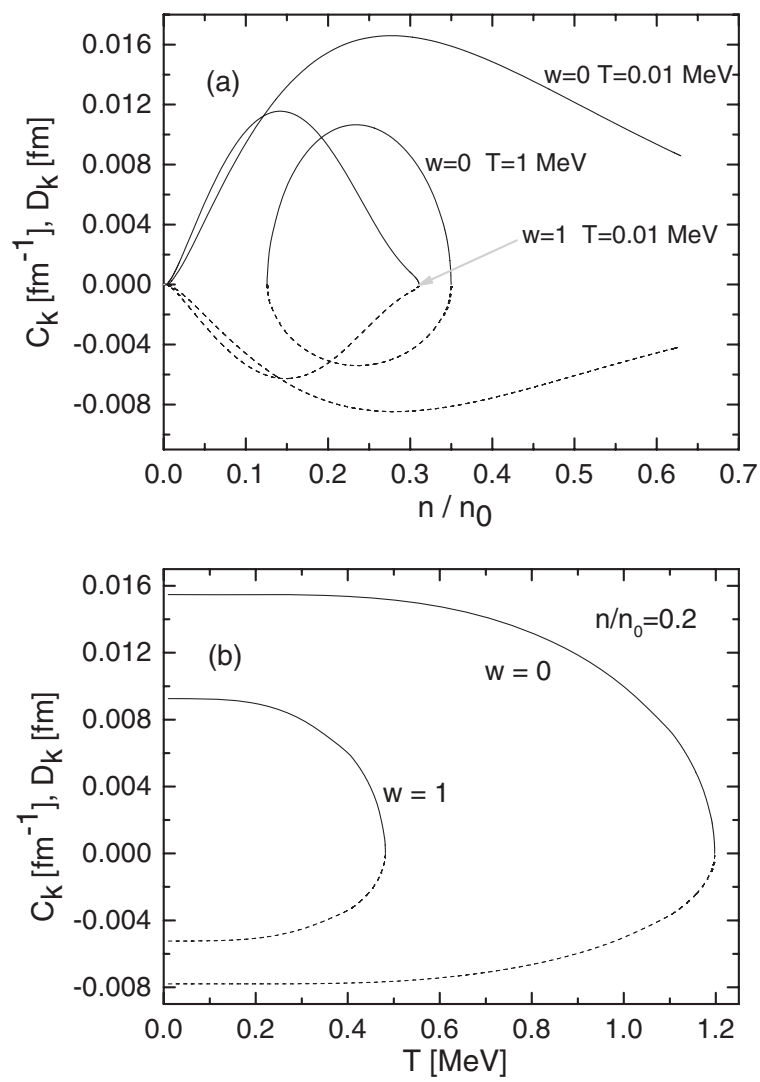

FIG. 10. The dependence of the gap coefficients $C_{k}$ and $D_{k}$ on the density (a) and temperature (b). In (a) each curve corresponds to fixed values of asymmetry and temperature as indicated by the labels. In (b) the density is fixed at $n / n_{0}=0.2$ for the asymmetries $w=0$ and 1 . In every situation $C$ (solid lines) and $D$ (dashed lines) appear as mirror-like curves.

component reaches, instead, the $w=1$ limit at low temperatures. But its isospin asymmetry extension is rapidly reduced as $T$ grows. The $T_{z}=1$ projection has a broader domain of $n<1.2 n_{0}, w<1$, and $T<2.6 \mathrm{MeV}$. The fact that this coupling survives up to supranormal densities and $w \sim 1$ has been noticed in reference to neutron star composition. For $T_{z}=0$ and $T_{z}=-1$ the upper temperature is reached in symmetric matter, whereas for $T_{z}=1$ it is attained at $w=0.8$.

The gap evaluated at the Fermi surface, $\Delta_{F}$, has a peak value slightly below the common results, although a comparison is hard to make due to the diversity of approaches and conclusions found in the literature. We obtained $\Delta_{F \max } \simeq$ $1.6 \mathrm{MeV}$ in symmetric matter and $\Delta_{F \max } \simeq 1.4$ in pure neutron matter. This comment is particularly true for $w=0$ at low temperature, while for $T_{z}=-1$ in neutron matter there is a clear resemblance to some elaborate calculations (see for instance [12]).

The $w$ dependence of the gap at the Fermi surface has distinguishing features for $T_{z}=1$ and $T_{z}=-1$. In the first case, $\Delta_{F 1} \rightarrow 0$ when $w \rightarrow 1$, whereas $\Delta_{F-1}$ keeps finite values at $w=1$. At low temperature there is a drastic change of regime according to the density value. A variation from decreasing in the low-density domain to a nonmonotonic behavior is registered in the $T_{z}=1$ channel. On the other 
hand, $\Delta_{F-1}$ changes from increasing, to nonmonotonic, to definitely decreasing as $n$ grows. For higher temperatures the range of accessible densities is shortened, and hence these modifications do not happen. We have found qualitative agreement with the results of Refs. [2,6] where a BCS calculation is performed based on the the AV18 model. It is worthwhile to mention that the inclusion of three-body forces in Ref. [2] improves the similarity.

The thermal behavior has the typical appearance for $T_{z}= \pm 1$ of a nonincreasing function with a steep fall at a characteristic temperature depending on the density and asymmetry of the system. For $T_{z}=0$ we found, at large enough $w$, the presence of a bivaluated critical temperature. This property has been previously described in relation to ${ }^{3} S_{1}-{ }^{3} D_{1}$ coupling [19].
Finally, we have examined the possibility of a BCS-BEC crossover at low temperature and $w=0$. But we do not find the typical change of sign of the effective chemical potential. Hence, we conclude that such a phenomenon cannot be properly described within this approach.

The present description can be improved in different ways. An analysis of the sensitivity to the cutoff procedure will be given, and the inclusion of effects of the liquid-gas instability is in progress.

\section{ACKNOWLEDGMENT}

This work was partially supported by CONICET, Argentina.
[1] A. I. Akhiezer, A. A. Isayev, S. V. Peletminsky, and A. A. Yatsenko, Phys. Rev. C 63, 021304(R) (2001).

[2] W. Zuo and G. C. Lu, Phys. Rev. C 75, 045806 (2007).

[3] J. Margueron, H. Sagawa, and K. Hagino, Phys. Rev. C 77, 054309 (2008).

[4] Y. Tian, Z. Y. Ma, and P. Ring, Phys. Lett. B 676, 44 (2009).

[5] K. Hebeler, T. Duguet, T. Lesinski, and A. Schwenk, Phys. Rev. C 80, 044321 (2009).

[6] S. S. Zhang, L. G. Cao, U. Lombardo, E. G. Zhao, and S. G. Zhou, Phys. Rev. C 81, 044313 (2010).

[7] N. Chamel, Phys. Rev. C 82, 014313 (2010).

[8] P. Ring and P. Schuck, The Nuclear Many-Body Problem (Springer-Verlag, Berlin, 1980).

[9] D. J. Dean and M. Hjorth-Jensen, Rev. Mod. Phys. 75, 607 (2003).

[10] A. I. Akhiezer, V. V. Krasil'nikov, S. V. Peletminsky, and A. A. Yatsenko, Phys. Rep. 245, 1 (1994); A. I. Akhiezer, A. A. Isayev, S. V. Peletminsky, A. P. Rekalo, and A. A. Yatsenko, Sov. Phys. JETP 85, 1 (1997).
[11] E. Khan, J. Margueron, G. Colo, K. Hagino, and H. Sagawa, Phys. Rev. C 82, 024322 (2010).

[12] L. G. Cao, U. Lombardo, and P. Schuck, Phys. Rev. C 74, 064301 (2006).

[13] M. Bender and P. H. Heenen, Rev. Mod. Phys. 75, 121 (2003).

[14] A. L. Fetter and J. D. Walecka, Quantum Theory of ManyParticle Systems (McGraw-Hill, New York, 1971).

[15] F. Douchin, P. Haensel, and J. Meyer, Nucl. Phys. A 665, 419 (2000).

[16] A. Fabrocini, S. Fantoni, A. Y. Illarionov, and K. E. Schmidt, Phys. Rev. Lett. 95, 192501 (2005); S. Gandolfi, A. Y. Illarionov, S. Fantoni, F. Pederiva, and K. E. Schmidt, ibid. 101, 132501 (2008).

[17] W. Zuo, U. Lombardo, H. J. Schulze, and C. W. Shen, Phys. Rev. C 66, 037303 (2002).

[18] M. Matsuo, Phys. Rev. C 73, 044309 (2006); J. Margueron, H. Sagawa, and K. Hagino, ibid. 76, 064316 (2007).

[19] A. Sedrakian, T. Alm, and U. Lombardo, Phys. Rev. C 55, R582 (1997); A. Sedrakian and U. Lombardo, Phys. Rev. Lett. 84, 602 (2000). 